\title{
Expression of Autophagy-Associated Genes in Skeletal Muscle: An Experimental Model of Chloroquine-Induced Myopathy
}

\author{
Noriyuki Kimura Toshihide Kumamoto Yosuke Kawamura Takahiro Himeno \\ Ken-ichro Nakamura Hidetsugu Ueyama Ryuki Arakawa \\ Department of Neurology and Neuromuscular Disorders, Faculty of Medicine, Oita University, Oita, Japan
}

\section{Key Words}

Apg12 - Apg $5 \cdot$ Autophagy $\cdot$ Chloroquine $\cdot \mathrm{GNE} \cdot \mathrm{LC} 3 \cdot$

Lysosome $\cdot \mathrm{mRNA} \cdot$ Rimmed vacuoles

\begin{abstract}
Objective: Chloroquine modulates autophagocytic protein degradation in the lysosome system, thereby inducing the formation of rimmed vacuoles consisting of autophagosomes and autolysosomes in skeletal muscle. The goal of this study was to investigate the contribution of the lysosomal system, particularly autophagosome formation (an autophagic process) at the molecular level, to the abnormal accumulation of vacuoles in an experimental model of chloroquine-induced myopathy. Methods: Histological, immunohistochemical and semiquantitative reverse transcriptase-polymerase chain reaction studies were performed on innervated and denervated rat soleus muscles after treatment with either saline or chloroquine. Results: Accumulation of rimmed vacuoles was observed only in chloroquine-treated denervated muscles. Microtubule-associated protein-1 light chain-3 (LC3) protein and mRNA levels were significantly increased exclusively in denervated muscles from chloroquine-treated rats, whereas Apg5 and Apg12 mRNA levels did not change significantly. Further, the mRNA levels of UDP-N-acetylglucosamine 2-epimerase/N-acetylmannosamine kinase (GNE), which are associated with distal
\end{abstract}

myopathy with rimmed vacuoles showing numerous rimmed vacuoles in its skeletal muscle, were not decreased in denervated muscles treated with chloroquine. Conclusions: LC3 mRNA may increase in association with rimmed vacuole formation in denervated muscles from chloroquine-treated rats, suggesting an increase in autophagy at the molecular level. Abnormal accumulation of rimmed vacuoles in this myopathy does not appear to be mediated by inhibition of autophagosome-related genes or GNE gene.

Copyright $\odot 2007$ S. Karger AG, Basel

\section{Introduction}

Chloroquine, a lysosomotrophic agent, modulates autophagic protein degradation in the lysosome system, thereby inducing the formation of rimmed vacuoles consisting of dense granular bodies and vacuoles including many variably sized and shaped autophagosomes in skeletal muscles [1-3]. We previously demonstrated an increased number of rimmed vacuoles in denervated chloroquine-treated rat muscle but not in innervated chloroquine-treated muscle or in innervated or denervated muscles of saline-treated rats [2]. Interestingly, while vacuoles were only increased in chloroquine-treated denervated muscles, there was no difference in lysosomal enzyme activity when comparing saline-treated and chlo-

\section{KARGER}

Fax +4161306 1234

E-Mail karger@karger.ch

www.karger.com
(C) 2007 S. Karger AG, Basel

$1015-2008 / 07 / 0743-0169 \$ 23.50 / 0$

Accessible online at:

www.karger.com/pat
Dr. Toshihide Kumamoto

Department of Neurology and Neuromuscular Disorders

Faculty of Medicine, Oita University, Idaigaoka 1-1, Hasama, Yufu

Oita 879-5593 (Japan)

Tel. +8197586 5814, Fax +8197586 6502, E-Mail kumagoro@med.oita-u.ac.jp 
roquine-treated denervated muscles. Since an autophagic-lysosome process mediates degradation of muscle fibers in denervated muscles [2], chloroquine-induced dysfunction of the autophagic-lysosome process could represent an excellent mechanistic explanation for vacuole formation.

The autophagic-lysosome system plays an important role in the degradation and turnover of intracellular proteins, lipids, glycogens and organelles in skeletal muscle $[4,5]$. While this pathway has been well characterized, it is not clear how chloroquine treatment affects this process. Furthermore, the pathomechanism of abnormal rimmed vacuole accumulation in those muscles remains unknown.

Recent studies have reported that Apg12, Apg5 and microtubule-associated protein-1 light chain-3 (LC3) are involved in the process of autophagy [6,7]. Indeed, autophagy-defective mutations (apg ant mut) in Apg12 and Apg5 result in abnormalities in autophagosome formation. Further, the Apg12p-conjugated Apg5p complex is essential for formation of the autophagosome precursor and final product $[6,7]$. This conjugation is found in various mammalian tissues, including skeletal muscle, and is thought to function in autophagy in the mammalian cell [6]. However, there is no direct evidence that the system is related to autophagy in rat skeletal muscle. In fact, the expression of the Apg12-conjugated Apg5 complex has only rarely been studied in skeletal muscle, especially under pathologic conditions.

Another ubiquitin-like modification, LC3, is also essential for the formation of the autophagosome [8-11]. LC3, a homologue of Apg8p/Aut7p in yeast, is associated with the autophagosome membrane after processing and plays a critical role in the formation of autophagosomes [12]. LC3 is present in various mammalian cells, including myocytes, as well as in yeast. Two forms of LC3, LC3-1 and -II, are produced posttranslationally in various cells. LC3-1 occurs in the cytoplasm, while LC3-II occurs in the autophagosome membrane. The amount of LC3-II correlated with the extent of autophagosome formation. LC3-II is the first identified mammalian protein to specifically associate with autophagosome membranes [13].

Rimmed vacuoles are frequently observed in the skeletal muscles of patients with distal myopathy with rimmed vacuoles, and UDP-N-acetylglucosamine 2-epimerase/ $\mathrm{N}$-acetylmannosamine kinase (GNE) gene mutations have been detected in this myopathy $[14,15]$. However, it is not clear whether or not chloroquine modulates GNE gene expression in skeletal muscle.
Therefore, the goal of this study was to investigate the contribution of the lysosomal system, particularly autophagosome formation (an autophagic process), and GNE expression to the abnormal accumulation of vacuoles in an experimental model of chloroquine-induced myopathy.

\section{Materials and Methods}

\section{Animals}

The left hind legs of 30 adult male Wistar rats (200-250 g) were denervated by ligation of the sciatic nerve, as previously described [2]. Chloroquine chloride (50 $\mathrm{mg} / \mathrm{kg}$ body weight) was injected intraperitoneally into 15 rats twice daily, beginning the day after denervation. The remaining 15 rats received injection of saline. The soleus muscles from the right (innervated) and left (denervated) legs were obtained from chloroquine- and saline-treated rats on days 2, 4 and 8 after the initial injection. The muscles were then rapidly frozen in isopentane cooled in liquid nitrogen.

\section{Histologic and Immunohistochemical Studies}

Routine histologic analysis was performed using cryostat sections (10- $\mu \mathrm{m}$ thick), as described previously [2]. Hematoxylineosin preparations of each specimen were analyzed with a Nikon Cosmozon ISA image apparatus (Nikon, Tokyo, Japan) attached to a Macintosh computer (Apple Computer, Cupertino, Calif., USA). The number of fibers with dense granular bodies or vacuoles at the light-microscopic level was determined in 400 muscle fibers per muscle.

For immunofluorescence analysis, the sections were incubated at room temperature for $2 \mathrm{~h}$ with a polyclonal goat antibody against the LC3 (diluted 1:50; Santa Cruz Biotechnology, Santa Cruz, Calif., USA), then with Texas red-labeled horse anti-goat IgG (diluted 1:100; Vector Laboratories, Burlingame, Calif., USA) for $1 \mathrm{~h}$, rinsed, and mounted on slides for visualization by fluorescence microscopy. Images were captured using a Carl Zeiss LSM5 Pascal-V3.2 confocal microscopy system (Carl Zeiss, Jena, Germany).

Immunostaining was specific because there was no staining when sections were allowed to react without the first-layer antibodies or when normal goat serum was substituted for the antibody.

\section{Semiquantitative Reverse Transcriptase Polymerase Chain} Reaction (RT-PCR)

Innervated and denervated soleus muscles from saline- and chloroquine-treated rats that had been histologically examined were excised and frozen rapidly in liquid nitrogen. Total RNA was isolated from the specimens with acid guanidinium thiocyanate buffer (Nippon Gene, Tokyo, Japan) according to the manufacturer's instructions. Complementary DNA (cDNA) was synthesized from $1 \mu \mathrm{g}$ of total RNA using 200 units of Moloney murine leukemia virus reverse transcriptase (Gibco, Gaithersburg, Md., USA) and $1 \mu \mathrm{g}$ of oligo-(dT) 12-18 primer (Invitrogen, Carlsbad, Calif., USA).

PCR primers were constructed on the basis of published nucleotide sequences of the rat LC3 gene [16] (5'-CA GGA TCC 

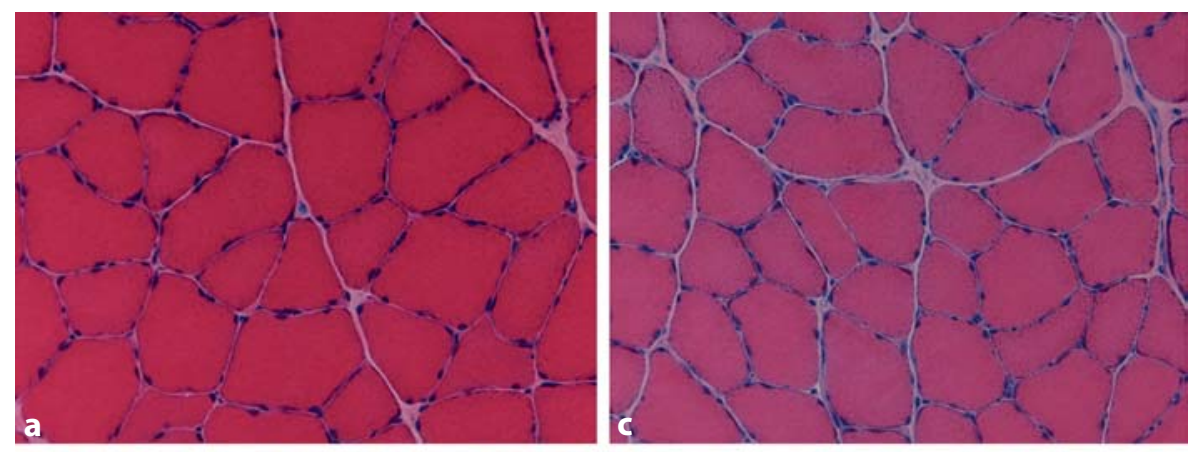

Fig. 1. Cross-sections of rat soleus muscles after 8 days of saline or chloroquine treatment. A large number of dense granular bodies and vacuoles are present in the denervated muscles from chloroquine-treated rats (d) but are absent in innervated muscles from chloroquine-treated rats (c) and in innervated (a) and denervated (b) muscles from saline-treated rats. HE. Bar $=50 \mu \mathrm{m}$.
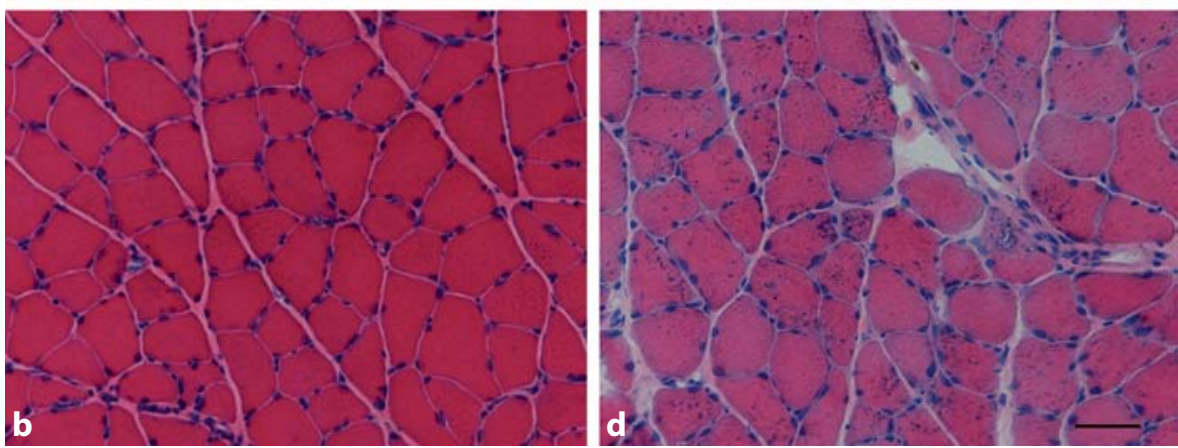

Fig. 2. Immunofluorescence staining for anti-LC3 antibody in innervated and denervated soleus muscles after 8 days of saline or chloroquine treatment. Immunoreactivity for LC3 was increased in the denervated muscles from chloroquinetreated rats (b) compared to the innervated saline-treated muscles (a). Bar $=50 \mu \mathrm{m}$.
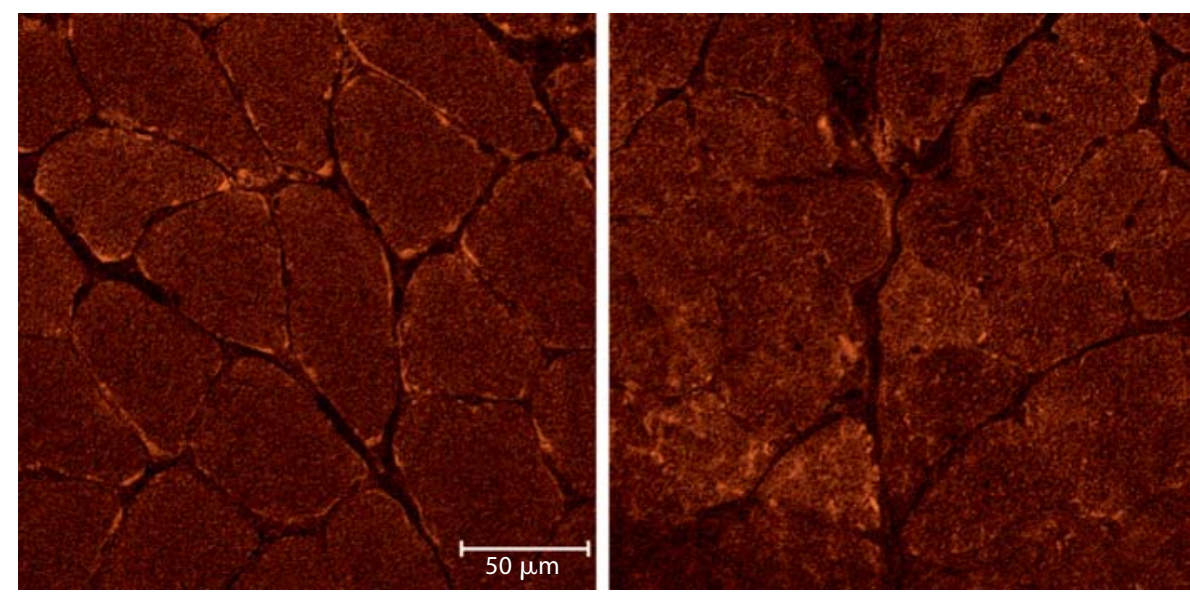

ATG CCG TCC CAG AAG ACC-3', sense; 5'-GTC CCT TTT TGC CTT GGT AG-3' antisense), the mouse Apg5 gene [7] (5'GCT TCG AGA TGT GTG GTT-3', sense; 5'-AAT GTA CTG TGA TGT TCC-3', antisense), the mouse Apg12 gene [7] (5'CCG GAG ACA CCA AGA AAA-3', sense; 5'-AGG GGC AAA GGA CTG ATT-3', antisense), the rat GNE gene [17] (5'-TGG TGG TGC TGG GCT CTC-3', sense; 5'-TGC TTA ATG TCG GTG GTC-3', antisense), and the rat glycerol aldehyde 3-phosphate dehydrogenase (GAPDH) gene [18] (5'-GCC AAG TTC AAT GGC ACA GT-3', sense; 5' - AAG GTG GAG GAA TGG GAG TT-3', antisense). The PCR reactions were performed in a final volume of $50 \mu \mathrm{l}$ in a DNA thermal cycler (Astec, Tokyo, Japan). Each PCR reaction mixture contained $1 \mu \mathrm{l}$ of $\mathrm{cDNA}, 10 \times$ PCR buffer (200 mM Tris- $\mathrm{HCl}, \mathrm{pH} 8.4$, and $500 \mathrm{mM} \mathrm{KCl}), 2.5 \mathrm{~mm}$ dNTP, $25 \mathrm{mM} \mathrm{MgCl}_{2}, 0.5 \mu \mathrm{M}$ of each set of primers, and 2.5 units of Taq DNA polymerase (Gibco). The PCR mixture was incubated at $94^{\circ} \mathrm{C}$ for 2 or $3 \mathrm{~min}$, followed by 28 or 35 cycles of amplification. Each cycle consisted of $30 \mathrm{~s}$ of denaturation at $94^{\circ} \mathrm{C}$, $30 \mathrm{~s}$ of annealing at 58 or $60^{\circ} \mathrm{C}$, and $30 \mathrm{~s}$ or $1 \mathrm{~min}$ of extension at $72^{\circ} \mathrm{C}$. After 28 or 35 cycles, a final extension step at $72^{\circ} \mathrm{C}$ for 3 min was performed. A negative control consisted of a reaction without inclusion of the reverse transcriptase. Efficiency of reverse transcription was controlled by PCR using GAPDH-specific primers. Target sequences of these primers were located on different exons. Only a proper reverse transcriptase reaction resulted in an amplification of a PCR product of the correct fragment size. After $2 \%$ agarose gel electrophoresis, scanning densitometry (Epson GT-8000 scanner; Epson, Tokyo, Japan) was used to determine the peak areas and assess the relative amounts of mRNA in ethidium bromide-stained bands. The intensity of 
Fig. 3. a Semiquantitative analysis of relative LC3 mRNA levels in innervated $(1,3)$ and denervated soleus muscles $(2,4)$ from rats treated with saline $(1,2)$ and chloroquine $(3,4)$ on days 2,4 , and 8 based on RT-PCR. b Changes in LC3 mRNA levels in innervated $(O, O)$ and denervated rat soleus muscles $(\square, \boldsymbol{\square})$ on days 2,4 , and 8 of saline $(\bigcirc, \square)$ or chloroquine treatment $(\mathbf{\square})$. Values are normalized to the LC3 mRNA level in innervated muscles of saline-treated rats (control muscles). Means \pm SD. $n=5$ rats per group.

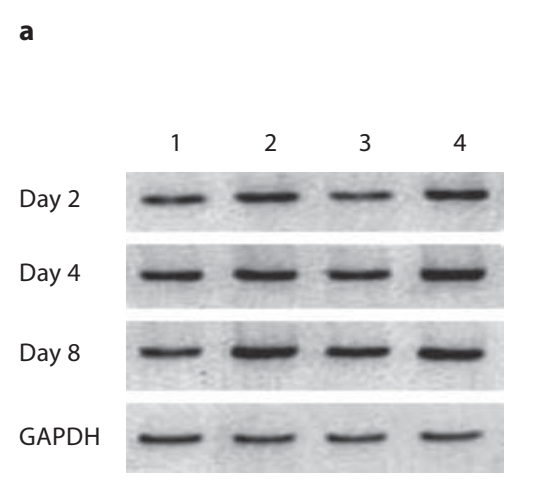

b

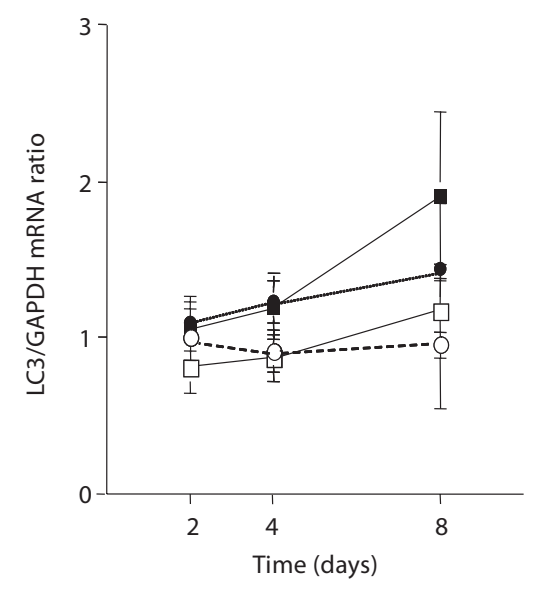

each band was quantified with NIH image (version 1.61) on a Macintosh computer. LC3, Apg5, Apg12, and GNE mRNA levels were normalized relative to GAPDH mRNA levels.

\section{Statistical Analysis}

Differences between control specimens and disease specimens were evaluated with a two-way analysis of variance. Multiple comparisons were made using Tukey's honestly significant difference test. A p value $<0.05$ was considered statistically significant.

\section{Results}

\section{Histologic Study}

Histologic findings for the innervated and denervated soleus muscles from saline- and chloroquine-treated rats on each test day were similar to those previously reported [2, 19] (fig. 1). Marked accumulation of dense granular bodies and vacuoles was present in chloroquine-treated muscles after denervation, particularly on day 8 . By contrast, accumulation was not noted in the contralateral, innervated chloroquine-treated muscles or in the innervated and denervated muscles of the saline-treated rats. Quantitative analysis at the light-microscopic level showed that the fibers with dense granular bodies or vacuoles were present in innervated and denervated muscles from rats after chloroquine treatment $(1.7 \pm 1.2$ and 12.8 $\pm 8.2 \%$ on day $2,9.9 \pm 1.7$ and $20.4 \pm 5.2 \%$ on day 4 , and $9.9 \pm 4.5$ and $52.2 \pm 15.0 \%$ on day 8 , respectively). Further, fiber numbers were significantly greater in the denervated muscles than in the contralateral, innervated muscles (on day 4, $\mathrm{p}=0.0006$; on day $8, \mathrm{p}<0.0001$ ). A few fibers with these bodies or vacuoles were found in the innervated and denervated muscles from saline-treated rats.

LC3 staining was minimal in innervated muscles from saline- and chloroquine-treated rats at all time points, whereas denervated muscles from both groups showed progressively stronger LC3 staining with increasing time (fig. 2). Strong positive reaction for LC3 was observed in the cytoplasm of muscle fibers, especially in the denervated muscles of chloroquine-treated rats.

\section{Semiquantitative RT-PCR}

To test for possible contamination of chromosomal DNA during RNA extraction, PCR amplification was performed without reverse transcription using the extracted RNA as a template. No bands appeared following 28 or 35 cycles of PCR, indicating the absence of contaminating DNA.

In the present study, LC3, Apg12 and Apg 5 mRNA levels were measured in the innervated and denervated soleus muscles of saline- and chloroquine-treated rats on 2-8 days after initial injection of chloroquine (fig. 3-5). Experiments revealed that LC3, Apg5, and Apg12 were constitutively expressed in those muscles.

Significant changes in LC3 mRNA levels occurred as early as day 2 in the denervated muscles from chloroquine-treated rats (fig. 3). Further, mRNA levels were increased in these muscles on all test days when compared with the innervated muscles from saline-treated rats (control muscles), with the respective levels on days 2,4 and 8 being 1.4, 1.6 and 1.8 times the control muscle levels, respectively. LC3 mRNA levels were significantly increased on days 4 and 8 in the denervated muscle of 
Fig. 4. a, c Semiquantitative analysis of relative Apg5 (a) and Apg12 (c) mRNA levels in innervated $(1,3)$ and denervated soleus muscles $(2,4)$ from saline $(1,2)$ - and chloroquine-treated rats $(3,4)$ on days 2,4 , and 8 based on RT-PCR. b, d Changes in Apg5 (b) and Apg12 (d) mRNA levels in innervated $(\bigcirc, 0)$ and denervated rat soleus muscles $(\square, \boldsymbol{\square})$ on days 2,4 , and 8 of saline $(\bigcirc, \square)$ or chloroquine treatment $(\boldsymbol{\square}, \mathbf{\square})$. Values are normalized to the Apg5 and Apg12 mRNA levels in innervated muscles of saline-treated rats (control muscles). Means \pm SD. $n=5$ rats per group.

Fig. 5. a Semiquantitative analysis of relative GNE mRNA levels in innervated $(1,3)$ and denervated soleus muscles $(2,4)$ from saline $(1,2)$ - and chloroquine-treated rats $(3,4)$ on days 2,4 , and 8 based on RT-PCR. b Changes in GNE mRNA levels in innervated $(\bigcirc, 0)$ and denervated rat soleus muscles $(\square, \boldsymbol{\square})$ on days 2,4 , and 8 of saline $(\bigcirc, \square)$ or chloroquine treatment $(\mathbf{\square}, \mathbf{\square})$. Values are normalized to the GNE mRNA level in innervated muscles of saline-treated rats (control muscles). Means \pm SD. $\mathrm{n}=5$ rats per group.
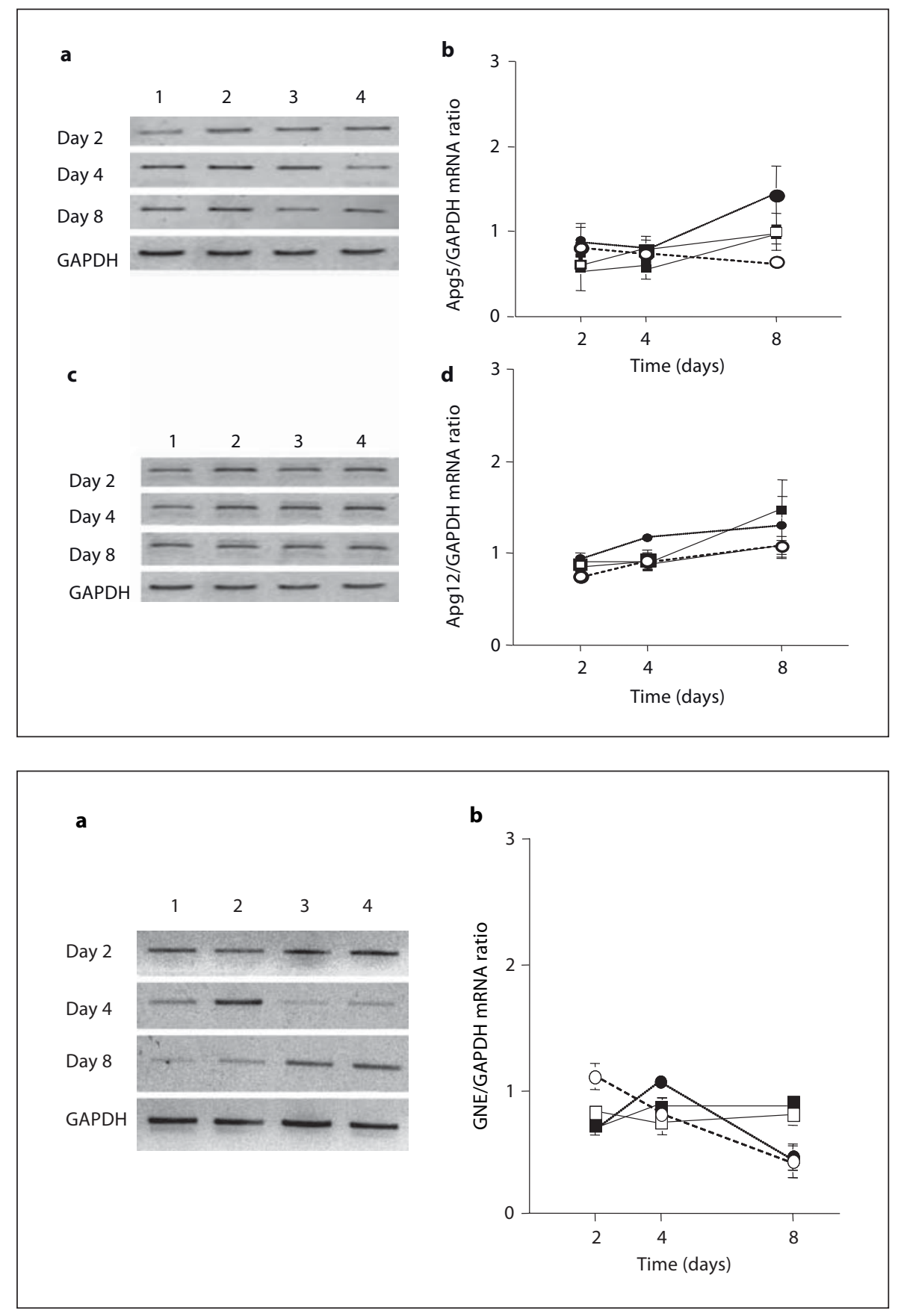

chloroquine-treated rats relative to those of the controls (on day $4, \mathrm{p}=0.051$; on day $8, \mathrm{p}=0.001$ ), but differences in mRNA levels on day 2 did not reach the levels of statistical significance. In the denervated muscles from saline-treated rats, LC3 mRNA levels progressively increased and were 1.1, 1.2 and 1.6 times those of control muscles, but the difference was not statistically significant. LC3 mRNA levels were increased in the denervat- ed muscles from chloroquine-treated rats as compared with the levels from the denervated muscles from saline-treated rats, but this difference was not statistically significant.

Apg5 and Apg12 mRNA levels in the denervated muscles from saline-treated rats were 0.9-, 1.1-, and 1.4-, and 1.1-, 1.1-, and 1.3-fold those of the control on days 2, 4, and 8 , whereas those in the denervated muscle from chlo- 
roquine-treated rats were $0.8-, 0.8-$, and $1.4-$, and 1.1-, $1.2-$, and 1.8-fold (fig. 4). However, no significant differences were found in the mRNA levels of Apg 5 and Apg12 when comparing between the denervated muscles of both groups on each test day.

GNE was constitutively expressed in the innervated and denervated muscles from saline- and chloroquinetreated rats (fig. 5). GNE mRNA expression tended to decrease in association with chloroquine treatment but not with denervation. Further, mRNA levels tended to increase in denervated, chloroquine-treated muscles during the experiment, but there was no statistically significant difference in mRNA levels in those muscles relative to the control muscles and denervated, saline-treated muscles.

\section{Discussion}

Previous studies have reported marked and early accumulation of rimmed vacuoles consisting of dense granular bodies and variously sized vacuoles in the denervated muscles of chloroquine-treated rats [1-3]. By contrast, these vacuoles were very rare in the contralateral, innervated chloroquine-treated muscles as well as in the innervated and denervated muscles of saline-treated rats. We previously reported significant increases in lysosomal enzyme (cathepsins B and L) activity and lysosome-related transport proteins, such as clathrin protein, and mRNA levels and mannose-6-phosphate receptor (M6PR) mRNA levels in the denervated muscles of chloroquine-treated rats compared with that in the innervated muscles of saline-treated rats $[1,2,19,20]$. These data suggest that the autophagic-lysosome system, especially generation of early endosomes, is increased, as manifested by the formation of lysosomal enzyme-containing clathrin-coated vesicles from the trans-Golgi network of the Golgi apparatus (an endosomal pathway) $[1,2,19,20]$. However, the autophagic process in these muscles has not yet been studied.

Both Apg5 and Apg12 are located only in the pre-autophagosomal structure (PAS), which precedes isolated membrane formation, and in the autophagosomal precursor but not in autophagosomes or autolysosomes [7, $13,21,22]$. LC3 is present mainly in autophagosomes and some autolysosomes as well as the autophagosomal precursor $[13,22]$. Autophagy is induced under various conditions. After breakdown of muscle fibers, segregation of cytoplasmic materials (e.g. proteins and lipids) and organelles (e.g. mitochondria and cell membranes) are surrounded with the membrane of autophagosomal precursor, and then autophagosomes are formed. The fusion of the outer membrane of autophagosomes with the membrane of early endosomes containing lysosomal enzymes then leads to the formation of autolysosomes [22]. In autolysosomes, segregated cytoplasmic materials or organelles are degraded by lysosomal enzymes, such as cathepsins B and L $[2,4]$. Although PAS is present in yeast [22], it is not clear whether PAS is present in animal cells.

In the present study, we measured mRNA levels of Apg12, Apg5 and LC3 in innervated and denervated muscles from saline- and chloroquine-treated rats and demonstrated expression of these genes in all experimental muscles. This suggests that the Apg12-conjugated Apg5 and LC3 systems may play an important role in physiological and pathological conditions in rat muscles. Previous reports have indicated that the Apg12-conjugated Apg5 system may be involved in autophagosome formation and completion in mammalian cells as well as in yeast cells $[6,7,21]$. However, the present study demonstrated that Apg12 and Apg5 mRNA levels were not significantly increased in denervated muscles of chloroquine-treated muscles, which have numerous dense granular bodies and vacuoles consisting mainly of autophagosomes compared with contralateral innervated muscles and with denervated and innervated muscles from saline-treated muscles.

The present study demonstrated that LC3 mRNA levels were increased in denervated muscles of chloroquinetreated rats relative to those in denervated muscles of saline-treated rats or innervated muscles of both rat groups from days 2-8. An increase in the formation of rimmed vacuoles containing dense granular bodies and vacuoles in denervated muscles of chloroquine-treated rats was observed as early as day 2 , with progressive increases thereafter. Further, the number of muscle fibers with these bodies and vacuoles increased along with the elevation of LC3 mRNA levels. These results suggest that LC3 may regulate autophagy and autophagosome formation in skeletal muscle and is consistent with previous in vitro data $[9,12,13,21]$. Therefore, chloroquine may induce autophagosome formation in the skeletal muscle without inhibiting LC3 expression.

In the present study, LC3 protein and mRNA levels significantly increased exclusively in denervated muscles from chloroquine-treated rats, whereas the mRNA levels of Apg12 and Apg5 did not change significantly. This discrepancy seems to be due to the fact that changes in Apg12 and Apg 5 mRNA levels are small. Further, it may be difficult to detect changes in Apg12 and Apg5 mRNA levels if the rate of turnover from PAS and the autophagosomal precursor to the autophagosome is very rapid. However, 
the nature of the interaction between the Apg12-Apg5 conjugate and LC3 at the transcriptional and molecular levels remains unclear.

The present and previous studies showed that mRNA or protein levels of major lysosome-related proteins, such as M6PR, clathrin, $\alpha$ - and $\gamma$-adaptins, Golgi-zone protein and LC3, were increased in the denervated muscles from chloroquine-treated rats that had numerous muscle fibers with rimmed vacuoles $[19,20]$. This suggests that the lysosome system, especially the transport of newly synthesized lysosomal enzymes from the secretory pathway via the trans-Golgi network and autophagosome formation, is activated in these muscles. However, the etiology and the pathomechanism of abnormal accumulation of rimmed vacuoles remain unclear. Recently, a GNE gene mutation has been detected in cases of familial inclusion body myopathy and distal myopathy with rimmed vacuoles $[14,15]$ associated with accumulation of rimmed vacuoles containing autophagosomes and autolysosomes, suggesting that a certain lysosome system abnormality may be responsible for rimmed vacuole formation. Although the expression of GNE in chloroquine-induced myopathy has not been studied previously, the present study demonstrated expression of GNE in innervated and denervated muscles from chloroquine- and saline- treated rats. However, skeletal muscle GNE mRNA levels did not change significantly after chloroquine or denervation treatments compared with control muscles. Thus, the role of GNE in the rimmed vacuole formation remains unclear.

In the present study, LC3 mRNA levels increased in association with rimmed vacuole formation in chloroquine-induced myopathy, suggesting an increase in autophagy at the molecular level. However, because of the expression of GNE, which may mediate formation of rimmed vacuoles in human skeletal muscles, we speculate that abnormal accumulation of rimmed vacuoles consisting of autophagosomes and autolysosomes in denervated muscles from chloroquine-treated rats may be due to slow turnover of autolysosomal degradation process at or after the fusion of early endosomes with autophagosomes. However, this process does not appear to be meditated by abnormalities in the early endosome or in autophagosome-related genes or proteins.

\section{Acknowledgments}

We are grateful to Ms. Y. Umeki, Ms. M. Ono, and Ms. K. Hirano for their technical assistance.

\section{References}

1 Kumamoto T, Araki S, Watanabe S, Ikebe N, Fukuhara N: Experimental chloroquine myopathy: morphological and biochemical studies. Eur Neurol 1989;29:202-207.

$\checkmark 2$ Kumamoto T, Ueyama $\mathrm{H}$, Watanabe $\mathrm{S}, \mathrm{Mu}-$ rakami T, Araki S: Effect of denervation on overdevelopment of chloroquine-induced autophagic vacuoles in skeletal muscles. Muscle Nerve 1993;16:819-826.

-3 Stauber WT, Hedge AM, Trout JJ, Schottelius BA: Inhibition of lysosomal function in red and white skeletal muscles by chloroquine. Exp Neurol 1981;71:295-306.

4 Bird JWC: Skeletal muscle lysosomes; in Dingle JT, Dean RT (eds): Lysosomes in Biology and Pathology. Amsterdam, North-Holland, 1975, vol 4, pp 75-109.

5 Gerard KW, Hipkiss AR, Schneider DL: Degradation of intracellular protein in muscle. Lysosomal response to modified proteins and chloroquine. J Biol Chem 1988;263: 18886-18890.

6 Mizushima N, Noda T, Ohsumi Y: Apg16p is required for the function of the Apg12pApg5p conjugate in the yeast autophagy pathway. EMBO J 1999;18:3888-3896.
7 Mizushima N, Yamamoto A, Hatano M, Kobayashi Y, Kabeya Y, Suzuki K, Tokuhisa T, Ohsumi Y, Yoshimori T: Dissection of autophagosome formation using Apg5-deficient mouse embryonic stem cells. J Cell Biol 2001;152:657-668.

8 Ichimura Y, Kirisako T, Takao T, Satomi Y, Shimonishi Y, Ishihara N, Mizushima N, Tanida I, Kominami E, Ohsumi M, Noda T, Ohsumi Y: A ubiquitin-like system mediates protein lipidation. Nature 2000;408:488492.

$\checkmark 9$ Nemoto T, Tanida I, Tanida-Miyake E, Minematsu-Ikeguchi N, Yokota M, Ohsumi M, Ueno T, Kominami E: The mouse APG10 homologue, an E2-like enzyme for Apg12p conjugation, facilitates MAP-LC3 modification. J Biol Chem 2003;278:39517-39526.

10 Ohsumi Y: Molecular dissection of autophagy: two ubiquitin-like systems. Nat Rev Mol Cell Biol 2001;2:211-216.
Tanida I, Sou YS, Ezaki J, Minematsu-Ikeguchi $\mathrm{N}$, Ueno $\mathrm{T}$, Kominami E: HsAtg4B/ HsApg4B/autophagin-1 cleaves the carboxyl termini of three human Atg8 homologues and delipidates microtubule-associated protein light chain 3- and GABAA receptor-associated protein-phospholipid conjugates. J Biol Chem 2004;279:36268-36276.

12 Kirisako T, Ichimura Y, Okada H, Kabeya Y, Mizushima N, Yoshimori T, Ohsumi M, Takao T, Noda T, Ohsumi Y: The reversible modification regulates the membrane-binding state of Apg8/Aut7 essential for autophagy and the cytoplasm to vacuole targeting pathway. J Cell Biol 2000;151:263-276.

13 Kabeya Y, Mizushima N, Ueno T, Yamamoto A, Kirisako T, Noda T, Kominami E, Ohsumi Y, Yoshimori T: LC3, a mammalian homologue of yeast Apg8p, is localized in autophagosome membranes after processing. EMBO J 2000;19:5720-5728.

14 Arai A, Tanaka K, Ikeuchi T, Igarashi S, Kobayashi H, Asaka T, Date H, Saito M, Tanaka H, Kawasaki S, Uyama E, Mizusawa H, Fukuhara N, Tsuji S: A novel mutation in the GNE gene and a linkage disequilibrium in Japanese pedigrees. Ann Neurol 2002;52: 516-529. 
15 Eisenberg I, Avidan N, Potikha T, Hochner $\mathrm{H}$, Chen M, Olender T, Barash M, Shemesh M, Sadeh M, Grabov-Nardini G, Shmilevich I, Friedmann A, Karpati G, Bradley WG, Baumbach L, Lancet D, Asher EB, Beckmann JS, Argov Z, Mitrani-Rosenbaum S: The UDP-N-acetylglucosamine 2-epimerase/ $\mathrm{N}$-acetylmannosamine kinase gene is mutated in recessive hereditary inclusion body myopathy. Nat Genet 2001;29:83-87.

- 16 Zhou B, Boudreau N, Coulber C, Hammarback J, Rabinovitch M: Microtubule-associated protein 1 light chain 3 is a fibronectin mRNA-binding protein linked to mRNA translation in lamb vascular smooth muscle cells. J Clin Invest 1997;100:3070-3082.
17 Stasche R, Hinderlich S, Weise C, Effertz K, Lucka L, Moormann P, Reutter W: A bifunctional enzyme catalyzes the first two steps in $\mathrm{N}$-acetylneuraminic acid biosynthesis of rat liver. Molecular cloning and functional expression of UDP-N-acetyl-glucosamine 2epimerase/N-acetylmannosamine kinase. J Biol Chem 1997;272:24319-24324.

18 Tso JY, Sun XH, Kao TH, Reece KS, Wu R: Isolation and characterization of rat and human glyceraldehyde-3-phosphatedehydrogenase cDNAs: genomic complexity and molecular evolution of the gene. Nucleic Acids Res 1985;13:2485-2502.

19 Masuda T, Ueyama H, Nakamura K, Jikumaru M, Toyoshima I, Kumamoto T: Skeletal muscle expression of clathrin and mannose 6-phosphate receptor in experimental chloroquine-induced myopathy. Muscle Nerve 2005;31:495-502.
20 Kumamoto T, Nagao SI, Sugihara R, Abe T, Ueyama H, Tsuda T: Effect of chloroquineinduced myopathy on rat soleus muscle sarcoplasm and expression of clathrin. Muscle Nerve 1998;21:665-668.

21 Mizushima N, Yamamoto A, Matsui M, Yoshimori T, Ohsumi Y: In vivo analysis of autophagy in response to nutrient starvation using transgenic mice expressing a fluorescent autophagosome marker. Mol Biol Cell 2004;15:1101-1111.

22 Suzuki K, Kirisako T, Kamada Y, Mizushima N, Noda T, Ohsumi Y: The pre-autophagosomal structure organized by concerted functions of APG genes is essential for autophagosome formation. EMBO J 2001;20: 5971-5981. 